\title{
Tocilizumab-induced pulmonary fibrosis in a patient with rheumatoid arthritis
}

\author{
Authors: Sanjeeva Silva, ${ }^{\mathrm{A}}$ Roshan Amarasena, ${ }^{\mathrm{A}} \mathrm{Jim}$ Moorcroft, ${ }^{\mathrm{B}}$ Tharshini Rajakulenthiran ${ }^{\mathrm{A}}$ and Ruchir Singh ${ }^{\mathrm{A}}$
}

\section{Introduction}

Interstitial lung disease (ILD) is a well-known extra-articular manifestation in patients diagnosed with rheumatoid arthritis (RA). The cardinal symptom of ILD is dyspnoea. There are several drugs which have been implicated in the development or exacerbation of ILD. Disease-modifying antirheumatic drugs (DMARDs) which cause drug-induced ILD are TNF alpha inhibitors and conventional DMARDs like methotrexate. However, interleukin 6 (IL-6) inhibitor-induced ILD is very rare and systematic literature revealed only a few cases of this type.

\section{Materials and methods}

This case report is about a patient with RA who developed severe acute pneumonitis after treatment with tocilizumab. An 82-year-old woman who had seropositive RA for 20 years was commenced on tocilizumab due to poor response to other DMARDs. She developed progressive worsening shortness of breath following the third infusion of tocilizumab. She was initially treated for possible community-acquired pneumonia and since there had been worsening of symptoms she was admitted to hospital. On admission she was dyspnoeic at rest and oxygen saturation was $88 \%$. Chest examination revealed bi-basal fine crepitation.

\section{Results and discussion}

Chest X-ray showed increased pulmonary interstitial markings throughout both lungs. Inflammatory markers were moderately high. Septic screening, including blood and sputum culture, was negative. She was treated with several intravenous antibiotics and her symptoms did not respond. Chest high-resolution computed tomography (CT) showed bilateral extensive predominantly subpleural honeycombing with traction bronchiectasis. She was commenced on oral prednisolone $40 \mathrm{mg}$ daily and her symptoms improved with steroid treatment. Tocilizumab was withheld and low-dose prednisolone continued. Serial chest X-rays showed resolution of interstitial markings and showed normal appearance following 10 months of the last tocilizumab dose. According to the literature, drug-induced ILD commonly develops within the first 20 weeks after initiation of therapy and old age is an added risk factor. ${ }^{1,2}$ Both features were seen in this case.

\section{Conclusion}

This case highlights the importance of considering druginduced ILD in patients with worsening of shortness of breath particularly of the new agents such as IL-6-inhibiting biologic DMARDs.

\section{Conflicts of interest}

None declared.

\section{References}

1 Roubille C, Haraoui B. Interstitial lung diseases induced or exacerbated by DMARDS and biologic agents in rheumatoid arthritis: a systematic literature review. Semin Arthritis Rheum 2014;43:613.

2 Singh JA, Cameron C, Noorbaloochi S et al. Risk of serious infection in biological treatment of patients with rheumatoid arthritis: a systematic review and meta-analysis. Lancet 2015;386:258. 